\section{Prevalência de sobrecarga e respectivos fatores associados em cuidadores de idosos dependentes, em uma região pobre do Rio de Janeiro, Brasil}

\author{
Prevalence of overburden in caregivers of \\ dependent elderly and associated factors in \\ a poor area of Rio de Janeiro, Brazil \\ Prevalencia de sobrecarga y factores asociados \\ en cuidadores de ancianos dependientes en una \\ región pobre de Río de Janeiro, Brasil
}

Valéria Teresa Saraiva Lino 1 Nadia Cristina Pinheiro Rodrigues 1 Luiz Antônio Bastos Camacho 1 Gisele O'Dwyer 1 Idenalva Silva de Lima 1

Mônica Kramer de Noronha Andrade 1 Soraya Atie 1

\begin{abstract}
Resumo
O envelhecimento populacional ocasionou aumento da dependência e da sobrecarga de cuidadores familiares de idosos dependentes. O objetivo foi verificar, entre cuidadores familiares, a prevalência de sobrecarga e os fatores associados a ela em uma região pobre e violenta do Rio de Janeiro, Brasil. Trata-se de estudo transversal com 140 idosos e cuidadores familiares, para investigar apoio social, maus tratos, coabitação e sobrecarga nos cuidadores familiares, além de dependência, declínio cognitivo e depressão no idoso. Modelos logísticos múltiplos foram construídos no intuito de explicar a sobrecarga dos cuidadores familiares. As seguintes características dos idosos se associaram à sobrecarga: idade (OR =0,94; $p<0,002)$, depressão $(O R=2,59 ; p<0,005)$ e declínio cognitivo $(O R=3,19 ; p<0,03)$. Em relação aos fatores dos cuidadores familiares, apenas apoio social manteve a relevância $(O R=2,35 ; p<0,005)$. Conclui-se que investigar e tratar depressão e demência em idosos, assim como prover apoio aos seus cuidadores, podem contribuir para o manejo efetivo da sobrecarga de cuidadores familiares, melhorando a qualidade do cuidado e a saúde de ambos.
\end{abstract}

Cuidadores; Idoso Fragilizado; Apoio Social

\author{
1 Escola Nacional de Saúde \\ Pública Sergio Arouca \\ Fundação Oswaldo Cruz, Rio \\ de Janeiro, Brasil. \\ Correspondência \\ V. T. S. Lino \\ Centro de Saúde Escola \\ Germano Sinval Faria, Escola \\ Nacional de Saúde Pública \\ Sergio Arouca, Fundação \\ Oswaldo Cruz. \\ Rua Leopoldo Bulhões 1480, \\ Rio de Janeiro, RJ 21041-210, \\ Brasil. \\ valerialino@ensp.fiocruz.br
}




\section{Introdução}

Aproximadamente 200 milhões de indivíduos ao redor do mundo apresentam dependência significativa para o desempenho de atividades cotidianas, situação com tendência crescente, em virtude do envelhecimento populacional e do aumento da prevalência de doenças crônicas. A maior concentração de idosos dependentes ocorre entre aqueles com 80 ou mais anos, grupo etário que mais cresce 1 . No Brasil, apesar dos níveis crescentes de incapacidade, ainda não conseguimos dimensionar o impacto sobre o sistema de saúde tanto da dependência, quanto da necessidade de cuidadores para os idosos dependentes. O reconhecimento do papel do cuidador, seja familiar, seja profissional, necessita ser considerado no planejamento de políticas públicas 2 .

A função de cuidador tem sido prestada por um "sistema" de suporte informal, que inclui amigos, vizinhos, mas, principalmente, os familiares 3 . Mulheres de meia idade predominam no exercício da função de cuidador, embora as mais idosas também assumam esta função. $\mathrm{O}$ baixo índice de escolaridade, os períodos prolongados dedicados ao cuidado e a ausência de revezamento na tarefa caracterizam o universo dos cuidadores familiares, havendo evidências de que eles apresentam pouco domínio sobre os problemas de saúde e também sobre os cuidados necessários demandados pelos idosos 4 .

A tarefa de cuidar acarreta, frequentemente, depressão e diminuição da qualidade de vida 4 , fatores que, aliados à ausência de serviços domiciliares e à coabitação com o idoso dependente, contribuem para o estresse emocional vivenciado no cotidiano. Entretanto, a principal fonte de sobrecarga é o grau de dependência do indivíduo que recebe o cuidado 5,6.

Elevada prevalência de estresse em cuidadores familiares de idosos já foi identificada no Brasil. Dois estudos realizados com amostras numerosas de cuidadores residentes no Paraná revelaram a prevalência de sobrecarga moderada a severa em mais de $70 \%$ dos familiares responsáveis pelo cuidado de idosos dependentes 7,8. Em São Paulo e na Paraíba, Yamashita et al. ${ }^{9}$ e Loureiro et al. 10 também identificaram a ocorrência de estresse em $69 \%$ e $84 \%$ dos cuidadores, respectivamente. Comparados com os achados desses estudos, os níveis de sobrecarga inferiores a $30 \%$ podem ser considerados reduzidos nos cuidadores examinados em São Paulo por Gratão et al. 6, numa pesquisa cuja prevalência de dependência foi de apenas $15 \%$.

O ônus acarretado pela atividade de cuidador tem implicações na ocorrência de violência (também referida como abuso ou maus tratos) familiar e no consumo problemático de álcool. A sobre- carga constitui-se em fator de risco para violência perpetrada por cuidadores de idosos dementes e também daqueles dependentes para as atividades de vida diária 11 . O comportamento abusivo ocorreu em mais de $30 \%$ dos familiares de idosos dementes em uma amostra representativa da comunidade do Reino Unido, sendo a violência psicológica por meio de agressões verbais a mais comum 12. Em nosso meio, $10 \%$ dos 322 idosos usuários da atenção primária de Niterói, Rio de Janeiro, relataram ter sofrido violência física por parte de familiares. Entre os fatores associados aos maus tratos, incluíam-se condições geradoras de dependência para o idoso, como distúrbio de memória e artrose/reumatismo 13. Já em Recife, Pernambuco, uma amostra semelhante de idosos apresentou prevalência de $21 \%$ de violência doméstica, sendo mais frequente entre as mulheres, naqueles que coabitavam com um número maior de indivíduos e nos dependentes para as atividades instrumentais da vida diária 14. A associação entre sobrecarga e o uso prejudicial de álcool por cuidadores tem sido pouco explorada; contudo, ela foi preditiva de problemas associados ao consumo de álcool em cuidadores residentes em Chicago, Estados Unidos 15.

A tensão associada à tarefa de cuidar de idosos dependentes pode ser amenizada pelo apoio social 16. Este conceito se relaciona à satisfação do indivíduo com a sua rede de relacionamentos, integrando as seguintes dimensões: apoio afetivo, emocional, material, de informação e interação social positiva 17 . A oferta de apoio material e emocional ao cuidador alivia a sobrecarga e melhora as condições de atenção ao idoso fragilizado 18. Por outro lado, a ausência de apoio social pode acarretar a persistência de sintomas de estresse, mesmo após alguns anos da morte do idoso dependente 19 .

Ainda que a oferta de serviços de apoio a pessoas com deficiência seja, de forma geral, insuficiente, ela é comum em países ricos, mas ainda se constitui em um conceito novo em regiões menos desenvolvidas 1. É necessário examinar de perto os fatores relacionados à sobrecarga de cuidadores para identificar aqueles em risco de desfechos adversos, assim como os alvos para potenciais intervenções, de modo a se preservar a saúde do cuidador e da família, melhorando a qualidade do cuidado.

O objetivo do presente estudo foi verificar a prevalência de sobrecarga de cuidadores familiares de idosos dependentes e os fatores associados a esta, na região de Manguinhos, Rio de Janeiro, Brasil, entre janeiro de 2013 e junho de 2014. Espera-se contribuir para o conhecimento das características associados à sobrecarga de cuidadores em uma região com predomínio de violência e 
vulnerabilidade socioambiental. Seus achados poderão auxiliar na elaboração de ensaios clínicos destinados à redução de estresse de familiares.

\section{Métodos}

\section{Tipo de estudo e amostra}

Estudo transversal realizado na região de Manguinhos. Trata-se de área com extrema vulnerabilidade socioambiental, dividida em subáreas, onde residiam aproximadamente 36 mil pessoas em 2011. Os domicílios, feitos em alvenaria, tinham uma média de 2,8 habitantes cada um; a maioria tinha apenas um cômodo; quase $60 \%$ não dispunham de água da rede não oficial e mais de $30 \%$ não estavam conectados à rede geral de esgoto. A renda média domiciliar girava em torno de 630 Reais por mês, pouco maior do que o salário mínimo vigente à época. Quanto à escolaridade, quase 50\% dos residentes haviam completado apenas o ensino fundamental 20. Com um Índice de Desenvolvimento Humano (IDH) de 0,726, o bairro de Manguinhos ocupava o 122o lugar entre os 126 bairros da cidade (WikiRio. IDH dos bairros da cidade do Rio de Janeiro. http://www.wikirio.com.br/IDH_dos_bair ros_da_cidade_do_Rio_de_Janeiro, acessado em 10/Mar/2015). Nessa área, as iniciativas para a redução da violência têm sido inviabilizadas em face da atuação de traficantes de drogas 21 .

A amostra, de conveniência, foi constituída por idosos de ambos os sexos, identificados pelos cadastros das equipes de saúde da família. Dos 679 idosos com sessenta anos ou mais, residentes em Manguinhos, selecionou-se uma amostra de conveniência de 140 idosos, cujo critério de escolha baseou-se na facilidade de acesso aos domicílios. Para esta captação, 866 domicílios foram visitados. Incluíram-se indivíduos com duas ou mais dependências para a realização das atividades de vida diária (AVD), de forma a selecionar aqueles cujos cuidadores teriam maior propensão ao estresse relacionado ao ato de cuidar, dada a associação entre dependência e sobrecarga de cuidadores 1 . Todos deveriam dispor de um cuidador familiar responsável pela supervisão e cuidado da maior parte das atividades relacionadas ao idoso. Excluíram-se os independentes ou que apresentavam apenas uma dependência para uma AVD.

\section{Procedimentos}

Os dados foram coletados entre janeiro de 2013 e junho de 2014. Um profissional de nível médio foi treinado para identificar a ocorrência de dependência para AVD nos idosos e, durante a primeira visita, deveria convidar a dupla para o estudo. A identificação se deu por meio da Escala de Independência em Atividades da Vida Diária (EIAVD), ou Escala de Katz 22, sendo utilizada a versão adaptada para o português 23 . As perguntas eram dirigidas ao idoso ou, nos casos de impedimento para se obter a resposta com este, ao cuidador. A EIAVD consta de seis itens biologicamente hierarquizados em termos de complexidade, que aferem, de forma independente, o desempenho nas seguintes atividades de autocuidado: alimentação, controle de esfíncteres, transferência, higiene pessoal, capacidade para se vestir e tomar banho. Esta última é a mais complexa, pois a atividade de tomar banho, geralmente a primeira a requerer assistência, pode não demandar muito auxílio; todavia, dependência implica a necessidade de assistência para realização das tarefas, e a simples presença de uma pessoa para observar o banho caracteriza dependência. Por isso, foram selecionados aqueles com duas ou mais dependências.

Após a identificação dos idosos dependentes, esclarecimento dos objetivos do estudo para o idoso e seu cuidador, além da coleta da assinatura de ambos no termo de consentimento, o profissional agendava a visita do pesquisador. Os idosos incapazes de entender o termo não o assinavam. Em seguida, uma assistente social experiente na utilização dos instrumentos do estudo aplicava os questionários ao idoso e/ou cuidador e registrava as respostas dos voluntários em um telefone celular, enviando-as, de forma automática, para um banco de dados através da rede 3G de telefonia celular. Concluído o envio, os dados permaneciam no aparelho, porém de forma criptografada, não permitindo o acesso às informações.

Os instrumentos utilizados para avaliação do idoso são descritos a seguir: (1) Escala de Katz - a EIAVD - foi aplicada novamente para confirmação dos critérios de inclusão referentes à dependência para duas AVD. Nesta escala, cada dependência é considerada um ponto, definindo-se os seguintes níveis de dependência de acordo com a pontuação: leve - menor do que três; moderado - três ou quatro; alto - cinco ou mais pontos. (2) MiniExame do Estado Mental (MEEM) - trata-se de teste de triagem cognitiva, cujos escores variam de 0 , que indica maior grau de comprometimento cognitivo, até um total máximo de 30 pontos, correspondendo à melhor capacidade cognitiva. Para distinguir os indivíduos com e sem declínio, foram utilizados os seguintes pontos de corte, considerando-se a escolaridade: analfabetos - 19/20; um a sete anos de estudo - 23/24; oito ou mais anos de estudo - 26/27 24,25,26. (3) Escala de Cornell de Depressão em Demência - aplicada ao cuidador nos casos de declínio cognitivo do idoso, destinase à identificação de sintomas depressivos neste 
indivíduo. A pontuação total na escala é determinada pela soma dos itens, até um total de 28 , havendo depressão provável quando a soma é maior do que 10 e depressão definida quando acima de 18 pontos 27,28. (4) Escala de Depressão Geriátrica (EDG) - aplicada ao idoso sem declínio cognitivo para rastreamento de depressão. Utilizou-se a forma reduzida com 15 perguntas, pontuadas de zero a um, considerando-se seis ou mais pontos indicativos de depressão 29,30.

$\mathrm{Na}$ entrevista com o cuidador, utilizaram-se os seguintes instrumentos: (1) Escala de Avaliação de Sobrecarga de Zarit 31,32 - avalia o impacto de doenças mentais e físicas, apontando o nível de sobrecarga emocional dos cuidadores. Compõe-se de 22 questões que apresentam cinco possibilidades de resposta, indicando a frequência com que o indivíduo se sente sobrecarregado em diferentes situações. Para cada alternativa, a pontuação varia de 0 (nunca) a 4 (quase sempre). Neste estudo, consideraram-se três níveis de sobrecarga: ausente ou leve (menor que 21); moderada (21-40) e severa (acima de 40 pontos). (2) Questionário CAGE (Cut Down/Annoyed/Guilty/Eye-opener Questionnaire) para rastreamento de alcoolismo - o instrumento contém oito perguntas intermediárias, não pontuadas, que são adicionadas às quatro específicas. Considera-se haver problemas com álcool quando os entrevistados respondem afirmativamente a um mínimo de duas questões específicas 33,34. (3) Escala de Apoio Social do Medical Outcomes Stu$d y$ (MOS) - conta com 19 questões que abrangem as cinco dimensões de apoio social (apoio afetivo, emocional, material, de informação e interação social positiva). A versão em português demonstrou boa consistência interna, estabilidade e validade de constructo 17,35 . A pontuação é definida de acordo com a frequência de apoio percebida pelo indivíduo em cada uma das dimensões, podendo variar de 0 (nunca) a 4 (sempre). Foi considerado apoio social insatisfatório (ASI) quando houve mais de $50 \%$ de itens de cada dimensão com respostas iguais a 0,1 (raramente) ou 2 (às vezes). Mesmo que as dimensões da escala sejam bastante correlacionadas, dificultando a distinção entre elas 35 , optou-se por avaliar separadamente a associação entre cada uma delas e a sobrecarga do cuidador. (4) Caregiver Abuse Screen (CASE) para rastreamento de violência de cuidadores contra idosos - compõe-se de oito questões, cada uma podendo receber 0 ou 1 ponto, considerando-se risco aumentado de violência uma pontuação a partir de 4 . Na presente pesquisa, foi utilizada a versão adaptada para o português 36,37 .

\section{Análise estatística}

Frequências e percentuais foram calculados para as variáveis categóricas (sobrecarga do cuidador, sexo, estado civil, escolaridade, coabitação, alcoolismo, violência, depressão, nível de dependência e apoio social material, afetivo, emocional, interação social, informação e geral), enquanto média e desvio padrão foram calculados para a variável numérica idade. Testes estatísticos (ANOVA e Kruskal-Wallis) foram realizados para comparar a distribuição das características avaliadas segundo status de sobrecarga do cuidador. As seguintes variáveis foram mensuradas nos idosos: idade (em anos), sexo (masculino e feminino), estado civil (solteiro/divorciado/separado, viúvo, casado), escolaridade (analfabeto, 1-4 anos de estudo, 5 anos ou mais de estudo), depressão (sim, não), déficit cognitivo (sim, não) e nível de dependência (leve, moderado, alto). As variáveis mensuradas nos cuidadores foram: idade (em anos), sexo (masculino e feminino), estado civil (solteiro/divorciado/separado, viúvo, casado), coabitação com o idoso (sim, não), problemas com álcool (sim, não), violência (sim, não), ASI afetivo (sim, não), ASI emocional (sim, não), ASI interação social (sim, não), ASI informações (sim, não), ASI material (sim, não), ASI total (sim, não) e sobrecarga (ausente/leve, moderada, severa).

Utilizando-se como variável resposta "sobrecarga do cuidador" (em três categorias ordinais), foram construídos seis modelos logísticos ordinais múltiplos com chances proporcionais do tipo acumulativo, para avaliar a relação da sobrecarga do cuidador com os fatores apoio social, declínio cognitivo, depressão, violência e idade. Para evitar problemas de colinearidade, cada um dos seis modelos incluiu apenas uma dimensão de apoio social ou o escore total de apoio social (dimensões: afetivo, emocional, interação social, informações, material e total). A escolha das variáveis explicativas do modelo se baseou na literatura 7,12,15,16,19 Optou-se pela utilização do modelo logístico ordinal, uma vez que este era capaz de incorporar a informação da ordenação das categorias de sobrecarga do cuidador (variável resposta) no processo de inferência. A ordenação natural da variável resposta é um pressuposto para utilização desta técnica de modelagem. A interpretação dos resultados para a sobrecarga do cuidador (ausente/leve, moderada e severa) refere-se à chance de sobrecarga, seja severa, seja moderada, em relação à categoria ausente/leve ${ }^{38}$. Todas as análises utilizaram nível de 5\% de significância. Para analisar os dados, utilizou-se o software R versão 3.1.2 (The $\mathrm{R}$ Foundation for Statistical Computing, Viena, Áustria; http://www.r-project.org). 


\section{Considerações éticas}

O projeto se guiou pelas recomendações estabelecidas pelo Conselho Nacional de Saúde, que regulamenta pesquisas com seres humanos, e foi encaminhado ao Comitê de Ética em Pesquisa (CEP) da Escola Nacional de Saúde Pública Sergio Arouca, Fundação Oswaldo Cruz (ENSP/ Fiocruz). Todos os sujeitos envolvidos foram devidamente esclarecidos por meio do Termo de Consentimento Livre e Esclarecido (TCLE). Os casos em que se identificou a ocorrência de sobrecarga foram encaminhados para a equipe de saúde da família, que conta com equipe multidisciplinar. O estudo foi aprovado e recebeu o número 271/11 no CEP.

\section{Resultados}

Participaram do estudo 140 duplas de idosos e cuidadores. Perdas de informações representaram $2 \%$ a $3 \%$ do total. O grupo de idosos era formado predominantemente por mulheres viúvas ou separadas, com escolaridade inferior a quatro anos. Mais da metade sofria de depressão e 87,6\% apresentavam declínio cognitivo (Tabela 1). No grupo de cuidadores, houve predomínio de mulheres de meia idade, das quais $70 \%$ coabitavam com os idosos e quase $60 \%$ evidenciaram sinais de sobrecarga moderada a alta. Aproximadamente um terço desses indivíduos apresentou ASI e risco aumentado para violência doméstica. Além disso, índices preocupantes de problemas com álcool foram identificados (Tabela 2).

A análise da relação entre sobrecarga e os outros indicadores do cuidador apontou que apenas apoio social, principalmente apoio material e interação social, além de violência, apresentaram associações significantes (valor de $\mathrm{p}<0,05$ ) (Tabela 3).

$\mathrm{Na}$ análise das características dos idosos, verificou-se relação significante entre depressão e sobrecarga do cuidador (Tabela 4). No entanto, no modelo ajustado, construído para estimar a associação entre as diferentes características de idosos e sobrecarga, identificou-se a influência da idade, humor depressivo e nível de cognição dos idosos sobre o estresse de seus cuidadores (Tabela 5). A chance de sobrecarga mostrou-se $6 \%$ menor para cada acréscimo de um ano de idade $(\mathrm{OR}=0,94$; valor de $\mathrm{p}<0,002)$. A chance de sobrecarga mostrou-se 2,59 vezes maior no idoso com depressão em relação ao não depressivo $(\mathrm{OR}=2,59$; valor de $\mathrm{p}<0,005)$ e 3,19 vezes maior no idoso com déficit cognitivo em relação ao que não tem déficit cognitivo $(\mathrm{OR}=3,19$; valor de $\mathrm{p}<0,03$ ). Quanto aos cuidadores, apenas apoio social manteve a relevância. A chance de sobrecarga mostrou-se 2,60 vezes maior no cuidador com interação social insatisfatória em relação à satisfatória $(\mathrm{OR}=2,60$; valor de $\mathrm{p}<0,005) ; 2,27$ vezes maior no cuidador com apoio emocional insatisfatório em relação ao satisfatório (OR = 2,27; valor de $\mathrm{p}<0,02$ ); 2,15 vezes maior no cuidador com apoio de informações insatisfatório em relação ao satisfatório $(\mathrm{OR}=2,15$; valor de $\mathrm{p}$ $<0,02)$ e 1,86 vez maior no cuidador com apoio material insatisfatório em relação ao satisfatório $(\mathrm{OR}=1,86$; valor de $\mathrm{p}<0,05)$. A análise de resíduos revelou ajuste satisfatório do modelo foi ajustado (Tabela 5).

\section{Discussão}

No presente estudo, a prevalência de níveis moderados a elevados de sobrecarga de cuidadores atingiu quase $60 \%$. Esta prevalência provavelmente foi superestimada, pois foram selecionados aqueles idosos cujos cuidadores teriam maior propensão ao estresse relacionado ao ato de cuidar. Mesmo assim, taxas ainda mais altas de estresse envolvendo cuidadores de diferentes classes sociais foram relatadas no Brasil por Nardi et al. 7 , Seima et al. 8 e Loureiro et al. ${ }^{39}$, em diferentes estudos. Oliveira \& D'Elboux 4 revisaram 76 trabalhos brasileiros e também identificaram índices elevados de sobrecarga de cuidadores, havendo maior ônus para aqueles que se encontravam em condições sociodemográficas desfavoráveis. Apesar de um pouco menor, o nível de sobrecarga identificado em estudos internacionais também é elevado, como o de recente inquérito da Organização Mundial da Saúde (OMS), envolvendo 19 países, no qual quase 44 mil cuidadores familiares de idosos dependentes foram questionados a respeito de sobrecarga 40 . Esses dados demonstram a periculosidade para a saúde de que se reveste a condição de cuidador, independentemente do grupo populacional e dos locais onde residem as pessoas. Embora o cuidado informal seja vital para a sociedade, o ônus imposto aos cuidadores familiares de idosos dependentes implica numa maior atenção por parte dos profissionais e gestores da saúde. O financiamento estatal a serviços formais de suporte é um elemento importante para reduzir a assistência informal 1 e a profissionalização de cuidadores poderia se inserir nesta perspectiva.

Neste trabalho, três fatores relacionados aos idosos apresentaram associação com a sobrecarga de cuidadores familiares de idosos dependentes: idade, declínio cognitivo e depressão. Sabese que o envelhecimento é fator predisponente para o aparecimento de doenças crônicas que podem acarretar incapacidade e necessidade de 
Características gerais dos idosos participantes * do estudo. Manguinhos, Rio de Janeiro, Brasil, 2013-2014.

\begin{tabular}{|c|c|}
\hline Características & Idosos \\
\hline Idade [média (DP)] & $80,5(10,0)$ \\
\hline Sem informação & 0 \\
\hline \multicolumn{2}{|l|}{ Sexo $[n(\%)]$} \\
\hline Feminino & $96(70,6)$ \\
\hline Masculino & $40(29,4)$ \\
\hline Sem informação & 4 \\
\hline \multicolumn{2}{|l|}{ Estado civil [n (\%)] } \\
\hline Solteiro/Divorciado/Separado & $17(12,4)$ \\
\hline Viúvo & $76(55,5)$ \\
\hline Casado & $44(32,1)$ \\
\hline Sem informação & 3 \\
\hline \multicolumn{2}{|l|}{ Escolaridade (anos) [n (\%)] } \\
\hline 0 & $49(35,8)$ \\
\hline $1-4$ & $58(42,3)$ \\
\hline 5 ou mais & $30(21,9)$ \\
\hline Sem informação & 3 \\
\hline \multicolumn{2}{|l|}{ Depressão [n (\%)] } \\
\hline Presente & $67(47,9)$ \\
\hline Ausente & $73(52,1)$ \\
\hline Sem informação & 0 \\
\hline \multicolumn{2}{|l|}{ Déficit cognitivo [n (\%)] } \\
\hline Presente & $120(87,6)$ \\
\hline Ausente & $17(12,4)$ \\
\hline Sem informação & 3 \\
\hline \multicolumn{2}{|l|}{ Nível de dependência [n (\%)] } \\
\hline Leve & $17(12,5)$ \\
\hline Moderado & $51(37,5)$ \\
\hline Alto & $68(50,0)$ \\
\hline Sem informação & 4 \\
\hline
\end{tabular}

DP: desvio padrão.

* Total de participantes $=140$.

cuidadores a longo prazo 1 . O grupo aqui estudado, com idade média elevada, insere-se nesta perspectiva. Quanto aos transtornos mentais, eles acarretam comumente mais sobrecarga do que os problemas físicos. Em pacientes dementes, a dependência gera estresse de forma crônica, havendo correlação entre o nível de hormônios relacionados ao estresse, o tempo de cuidado, o estágio da doença, a presença de alterações de comportamento e o ato de cuidar ${ }^{41}$. Junte-se a esses fatores o despreparo dos familiares e o ônus financeiro gerado pela dependência, e está estabelecido um ambiente propício para o surgimento de sobrecarga ${ }^{42}$. Apesar disso, não existe na rotina médica, durante a consulta de um idoso demente, o hábito de questionar o cuidador sobre seu nível de es- tresse. Considerando-se a prolongada exposição da maioria dos cuidadores a demandas físicas e psicológicas, é natural a ocorrência de sobrecarga à medida que a doença avança. Apesar de grupos de apoio produzirem benefícios para a redução de depressão e melhoria do bem-estar psicológico de cuidadores de idosos dementes, seu efeito é menor no que tange à sobrecarga 42 , sugerindo a necessidade de outras medidas para manejo mais efetivo do estresse relacionado a esse tipo de cuidado.

Quase metade dos idosos do presente estudo apresentou depressão $(47,9 \%)$, indicando prevalência elevada no grupo. Asejulgar pela capacidade funcional e cognitiva da maioria dos participantes, pode-se compará-los a indivíduos residentes em instituições de longa permanência, entre os quais 
Tabela 2

Características gerais dos cuidadores participantes * do estudo. Manguinhos, Rio de Janeiro, Brasil, 2013-2014.

\begin{tabular}{|c|c|}
\hline Características & $n(\%)$ \\
\hline Idade [média (DP)] & $55,0(14,1)$ \\
\hline Sem informação & 0 \\
\hline \multicolumn{2}{|l|}{ Sexo } \\
\hline Feminino & $114(83,8)$ \\
\hline Masculino & $22(16,2)$ \\
\hline Sem informação & 4 \\
\hline \multicolumn{2}{|l|}{ Estado civil } \\
\hline Solteiro/Divorciado/Separado & $58(42,3)$ \\
\hline Viúvo & $12(8,8)$ \\
\hline Casado & $67(48,9)$ \\
\hline Sem informação & 3 \\
\hline \multicolumn{2}{|l|}{ Coabitação com idosos } \\
\hline Presente & $98(70,0)$ \\
\hline Ausente & $42(30,0)$ \\
\hline Sem informação & 0 \\
\hline \multicolumn{2}{|l|}{ Problemas com álcool } \\
\hline Presente & $25(18,2)$ \\
\hline Ausente & $112(81,8)$ \\
\hline Sem informação & 3 \\
\hline \multicolumn{2}{|l|}{ Violência } \\
\hline Presente & $46(33,6)$ \\
\hline Ausente & $91(66,4)$ \\
\hline Sem informação & 3 \\
\hline \multicolumn{2}{|l|}{ Sobrecarga } \\
\hline Ausente ou leve & $56(40,9)$ \\
\hline Moderada & $57(41,6)$ \\
\hline Severa & $24(18,2)$ \\
\hline Sem informação & 3 \\
\hline \multicolumn{2}{|l|}{ Apoio social insatisfatório } \\
\hline \multicolumn{2}{|l|}{ Afetivo } \\
\hline Presente & $20(14,6)$ \\
\hline Ausente & $117(85,4)$ \\
\hline Sem informação & 3 \\
\hline \multicolumn{2}{|l|}{ Emocional } \\
\hline Presente & $47(34,3)$ \\
\hline Ausente & $90(66,7)$ \\
\hline Sem informação & 3 \\
\hline \multicolumn{2}{|l|}{ Interação social } \\
\hline Presente & $53(38,7)$ \\
\hline Ausente & $84(61,3)$ \\
\hline Sem informação & 3 \\
\hline \multicolumn{2}{|l|}{ Informações } \\
\hline Presente & $51(37,2)$ \\
\hline Ausente & $86(62,8)$ \\
\hline Sem informação & 3 \\
\hline \multicolumn{2}{|l|}{ Material } \\
\hline Presente & $54(39,4)$ \\
\hline Ausente & $83(66,6)$ \\
\hline Sem informação & 3 \\
\hline \multicolumn{2}{|l|}{ Total } \\
\hline Presente & $40(29,2)$ \\
\hline Ausente & $97(70,3)$ \\
\hline Sem informação & 3 \\
\hline
\end{tabular}

DP: desvio padrão.

* Total de participantes $=140$.

Nota: o percentual foi calculado apenas para os casos válidos (com informação sobre os mesmos). 
Descrição percentual das principais características do cuidador segundo status de sobrecarga do cuidador. Manguinhos Rio de Janeiro, Brasil, 2013-2014.

\begin{tabular}{|c|c|c|c|c|}
\hline \multirow[t]{2}{*}{ Característica } & \multicolumn{3}{|c|}{ Sobrecarga } & \multirow[t]{2}{*}{ Valor de $\mathrm{p}$} \\
\hline & Ausente ou leve & Moderada & Severa & \\
\hline Idade [média (DP)] & $56,23(13,25)$ & $54,74(14,66)$ & $53,08(15,38)$ & 0,64 \\
\hline \multicolumn{5}{|l|}{ Sexo [n (\%)] } \\
\hline Feminino & $43(78,2)$ & $49(86,0)$ & $22(95,7)$ & 0,15 \\
\hline Masculino & $12(21,8)$ & $8(14,0)$ & $1(4,3)$ & \\
\hline \multicolumn{5}{|l|}{ Estado civil [n (\%)] } \\
\hline Solteiro/Divorciado/Separado & $26(46,4)$ & $23(40,4)$ & $9(37,5)$ & 0,93 \\
\hline Viúvo & $5(8,9)$ & $4(7,0)$ & $30(52,6)$ & \\
\hline Casado & $9(37,5)$ & $3(12,5)$ & $12(50,0)$ & \\
\hline \multicolumn{5}{|l|}{ Coabitação [n (\%)] } \\
\hline Presente & $42(75,0)$ & $38(66,7)$ & $18(75,0)$ & 0,57 \\
\hline Ausente & $14(25,0)$ & $19(33,3)$ & $6(25,0)$ & \\
\hline \multicolumn{5}{|l|}{ Alcoolismo [n (\%)] } \\
\hline Presente & $10(17,9)$ & $12(21,1)$ & $3(12,5)$ & 0,70 \\
\hline Ausente & $46(82,1)$ & $45(78,9)$ & $21(87,5)$ & \\
\hline \multicolumn{5}{|l|}{ Violência [n (\%)] } \\
\hline Presente & $7(12,5)$ & $22(38,6)$ & $17(70,8)$ & 0,01 \\
\hline Ausente & $49(87,5)$ & $35(61,4)$ & $7(29,2)$ & \\
\hline \multicolumn{5}{|l|}{ Apoio social insatisfatório [n (\%)] } \\
\hline \multicolumn{5}{|l|}{ Afetivo } \\
\hline Presente & $5(8,9)$ & $9(15,8)$ & $6(25,0)$ & 0,15 \\
\hline Ausente & $51(91,1)$ & $48(84,2)$ & $18(75,0)$ & \\
\hline \multicolumn{5}{|l|}{ Emocional } \\
\hline Presente & $13(23,2)$ & $24(42,1)$ & $10(41,7)$ & 0,07 \\
\hline Ausente & $43(76,8)$ & $33(57,9)$ & $14(58,3)$ & \\
\hline \multicolumn{5}{|l|}{ Interação social } \\
\hline Presente & $15(26,8)$ & $24(42,1)$ & $14(58,3)$ & 0,02 \\
\hline Ausente & $41(73,2)$ & $33(57,9)$ & $10(41,7)$ & \\
\hline \multicolumn{5}{|l|}{ Informações } \\
\hline Presente & $16(28,6)$ & $22(38,6)$ & $13(54,2)$ & 0,09 \\
\hline Ausente & $40(71,4)$ & $35(61,4)$ & $11(45,8)$ & \\
\hline \multicolumn{5}{|l|}{ Material } \\
\hline Presente & $17(30,4)$ & $22(38,6)$ & $15(62,5)$ & 0,03 \\
\hline Ausente & $39(69,6)$ & $35(61,4)$ & $9(37,5)$ & \\
\hline \multicolumn{5}{|l|}{ Total } \\
\hline Presente & $11(19,6)$ & $18(31,6)$ & $11(45,8)$ & 0,05 \\
\hline Ausente & $43(80,4)$ & $39(68,4)$ & $13(54,2)$ & \\
\hline
\end{tabular}

DP: desvio padrão.

Nota: os testes estatísticos ANOVA e Kruskal-Wallis foram utilizados para comparar as características segundo status de sobrecarga do cuidador.

a prevalência de depressão pode ser ainda mais elevada. Castro-de-Araújo et al. ${ }^{43}$, em uma revisão com metanálise de estudos brasileiros, identificaram a ocorrência de sintomas depressivos clinicamente significativos em $65 \%$ de idosos institucionalizados, havendo associação entre depressão, sexo feminino, idade e demência. Tendo em vista a já estabelecida relação entre transtorno de humor depressivo e incapacidade 44,45 , a presença de todas essas características contribuem para justificar as taxas elevadas de depressão encontradas nesta pesquisa, assim como sua associação com 
Descrição percentual das principais características do idoso segundo status de sobrecarga do cuidador.

Manguinhos, Rio de Janeiro, Brasil, 2013-2014.

\begin{tabular}{|c|c|c|c|c|}
\hline \multirow[t]{2}{*}{ Característica } & \multicolumn{3}{|c|}{ Sobrecarga } & \multirow[t]{2}{*}{ Valor de $p$} \\
\hline & Ausente ou leve & Moderada & Severa & \\
\hline Idade [média (DP)] & $79,96(16,25)$ & $81,34(8,94)$ & $75,54(12,35)$ & 0,13 \\
\hline \multicolumn{5}{|l|}{ Sexo [n (\%)] } \\
\hline Feminino & $37(69,8)$ & $44(78,6)$ & $13(54,2)$ & 0,08 \\
\hline Masculino & $16(30,2)$ & $12(21,4)$ & $11(45,8)$ & \\
\hline \multicolumn{5}{|l|}{ Escolaridade (anos) [n (\%)] } \\
\hline 0 & $25(47,2)$ & $19(33,3)$ & $5(20,8)$ & 0,17 \\
\hline $1-4$ & $20(37,7)$ & $24(42,1)$ & $11(45,8)$ & \\
\hline 5 ou mais & $8(15,1)$ & $14(24,6)$ & $8(33,3)$ & \\
\hline \multicolumn{5}{|l|}{ Estado civil [n (\%)] } \\
\hline Solteiro/Divorciado/Separado & $9(17,0)$ & $4(7,0)$ & $4(16,7)$ & 0,31 \\
\hline Viúvo & $29(54,7)$ & $35(61,4)$ & $9(37,5)$ & \\
\hline Casado & $15(28,3)$ & $18(31,6)$ & $11(45,8)$ & \\
\hline \multicolumn{5}{|l|}{ Depressão [n (\%)] } \\
\hline Presente & $17(30,4)$ & $33(57,9)$ & $15(62,5)$ & 0,01 \\
\hline Ausente & $39(69,6)$ & $24(42,1)$ & $9(37,5)$ & \\
\hline \multicolumn{5}{|l|}{ Déficit cognitivo [n (\%)] } \\
\hline Presente & $44(83,0)$ & $53(93,0)$ & $22(91,7)$ & 0,27 \\
\hline Ausente & $9(17,0)$ & $4(7,0)$ & $2(8,3)$ & \\
\hline \multicolumn{5}{|l|}{ Nível de dependência [n (\%)] } \\
\hline Leve & $5(8,9)$ & $7(12,3)$ & $5(23,8)$ & 0,19 \\
\hline Moderado & $19(33,9)$ & $26(45,6)$ & $5(23,8)$ & \\
\hline Alto & $32(57,1)$ & $24(42,1)$ & $11(52,4)$ & \\
\hline
\end{tabular}

DP: desvio padrão.

Nota: os testes estatísticos ANOVA e Kruskal-Wallis foram utilizados para comparar as características segundo status de sobrecarga do cuidador.

a sobrecarga dos cuidadores familiares de idosos dependentes, identificada de forma independente em relação aos outros fatores. Não obstante se dar mais atenção ao estresse ocasionado por demência, câncer e outras doenças causadoras de dependência física, a depressão é sabidamente um fator preditor de sobrecarga para os cuidadores $46 \mathrm{em}$ face da redução do funcionamento, o que dificulta a manutenção da independência no cotidiano. O ônus financeiro, social e relacionado à saúde ocasionado pelo ato de cuidar de idosos deprimidos acarreta prejuízo a longo prazo para seus cuidadores, que precisam ser incluídos nas intervenções voltadas para o tratamento da doença 47 .

Este trabalho identificou que, entre os fatores relacionados ao cuidador, apenas a variável "baixo apoio social" demonstrou associação com sobrecarga, confirmando os efeitos deletérios da dependência sobre os cuidadores familiares de idosos dependentes. O desafio de tratar adequadamente do idoso pode levar os cuidadores a experimentarem isolamento social, que é, por si só, um fator de risco e uma consequência da sobrecarga, transformando-os em "pacientes invisíveis" 48. Por outro lado, o apoio social é um fator determinante na redução da sobrecarga de cuidadores de indivíduos com doenças degenerativas 9 . Drentea et al. ${ }^{49}$ verificaram que cuidadores submetidos a uma intervenção para ajudá-los a mobilizar a rede de apoio social relataram maiores níveis de satisfação nos primeiros cinco anos de intervenção do que aqueles do grupo-controle. Apoio emocional, sensação de intimidade com outros e visitas mais frequentes foram preditivos de alterações longitudinais na satisfação com a rede de apoio social. No Brasil, Moraes \& Silva 42 verificaram que o tempo de cuidado diário e a obtenção de ajuda para a realização do cuidado apresentaram associação com a sobrecarga de cuidadores familiares de idosos dependentes de 
Associação entre as características dos idosos e cuidadores com sobrecarga. Manguinhos, Rio de Janeiro, Brasil, 2013-2014.

\begin{tabular}{lcc}
\hline Características & Sobrecarga do cuidador * & IC $95 \%$ \\
& OR & $0,91-0,98$ \\
Características do idoso & & $0,18-1,35$ \\
Idade (anos) & 0,94 & $1,27-5,39$ \\
Alto nível de dependência & 0,50 & $1,02-10,85$ \\
Depressão & 2,59 & $0,78-5,61$ \\
Déficit cognitivo & 3,19 & $1,09-4,78$ \\
Características do cuidador & & $1,29-5,38$ \\
Apoio social insatisfatório & & $1,04-4,49$ \\
Afetivo & 2,08 & $0,91-3,80$ \\
Emocional & 2,27 & $1,11-5,06$
\end{tabular}

IC95\%: intervalo de 95\% de confiança; OR: odds ratio.

* Foram construídos seis modelos logísticos ordinais com chances proporcionais do tipo acumulativo. Variável resposta: sobrecarga do cuidador. Fatores preditores incluídos nos modelos: idade, depressão, alto nível de dependência e déficit de cognição, além de apoio social ao cuidador. Cada um dos seis modelos incluiu apenas uma das dimensões de apoio social listadas na tabela: afetivo, emocional, interação social, informações, material e total. Foram estimadas OR para as variáveis idade, déficit cognitivo, alto nível de dependência e depressão com base no modelo ajustado pelo escore total de apoio social.

Nota: todas as variáveis categóricas do cuidador e do idoso são dicotômicas ("sim", "não"), sendo a categoria de referência representada pela categoria "não".

pacientes com doença de Alzheimer, evidenciando a importância do apoio social no cuidado do indivíduo demente. Tomomitsu et al. 50 examinaram os fatores relacionados à satisfação com a vida em cuidadores familiares de idosos dependentes de três cidades brasileiras e encontraram uma maior proporção de indivíduos satisfeitos e menos estressados no grupo com alto nível de apoio social.

Com relação às diferentes dimensões de apoio social examinadas neste trabalho, nota-se maior impacto da interação social positiva e do apoio emocional na sobrecarga de cuidadores. A alta correlação entre as dimensões da escala aqui utilizada 35 torna possível a investigação da percepção de apoio social em cuidadores com um número menor de dimensões.

Problemas graves com álcool foram identificados em 18\% dos cuidadores investigados nesta pesquisa, não tendo sido evidenciada associação com sobrecarga. Todavia, essa taxa é maior do que a evidenciada em outros grupos 51,52. Sabendo-se que estresse crônico pode desencadear comportamentos de risco relacionados à saúde, como o abuso de álcool 53, é oportuno incluir os cuidadores familiares de idosos dependentes nas políticas voltadas para a redução do consumo de bebida alcoólica.

O presente estudo também não identificou associação entre coabitação e sobrecarga, fator comumente causador de estresse em cuidadores familiares de idosos dependentes, cuja saúde comumente piora quando eles residem junto ao idoso 54 . A grande proporção de coabitação, somada ao reduzido tamanho da amostra, pode ter contribuído para a diminuição do poder estatístico, prejudicando a identificação daquela associação.

Esta pesquisa apresenta algumas limitações. Primeiro, ela é derivada de um estudo transversal, não sendo possível concluir-se a existência de associação causal entre a sobrecarga de cuidadores e os outros fatores aqui mencionados, factível em desenhos longitudinais. Como a amostra utilizada foi de conveniência, não é possível a generalização externa dos achados. Não identificamos o tempo gasto diariamente com o cuidado, sabidamente um fator de risco para sobrecarga. Também não há dados sobre o nível educacional dos cuidadores, sabendo-se que o baixo índice de escolaridade pode ter um impacto direto 
sobre o cuidado, em decorrência das dificuldades na compreensão do processo de adoecimento do idoso e da falta de acesso a informações, podendo gerar sobrecarga 6 . Entretanto, a despeito dessas limitações, este trabalho apresenta pontos fortes. É um estudo de base populacional, cujo tamanho amostral possibilitou a investigação de associação entre sobrecarga do cuidador e outras variáveis relacionadas ao próprio cuidador e também ao idoso dependente. As avaliações utilizaram instrumentos padronizados, já adaptados à nossa cultura. O fato de a região de Manguinhos apresentar um dos mais baixos IDH do Rio de Janeiro e de apenas metade dos moradores ter concluído o ensino fundamental nos permite inferir que a escolaridade dos cuidadores entrevistados é semelhante à dos outros moradores da localidade.

\section{Colaboradores}

V. T. S. Lino participou da concepção, elaboração e redação do artigo. N. C. P. Rodrigues e L. A. B. Camacho contribuíram na análise e interpretação dos dados, redação do artigo e aprovação final da versão a ser publicada. G. O'Dwyer, I. S. Lima e S. Atie colaboraram na concepção do artigo, revisão crítica do conteúdo intelectual e aprovação da versão final. M. K. N. Andrade contribuiu na redação do artigo e aprovação final da versão a ser publicada.

\section{Conclusão}

O envelhecimento acarreta o aumento do número de idosos dependentes, destacando-se a importância cada vez maior de serviços que envolvem o domicílio como esfera de atenção, onde os cuidadores familiares assumem papel relevante. Este estudo identificou o declínio cognitivo e a depressão em idosos como fatores associados ao estresse dos familiares que prestam o cuidado. Da mesma forma, verificou que o baixo nível de apoio social acarreta sobrecarga aos cuidadores. De posse desse conhecimento, futuros estudos visando à intervenção naqueles fatores poderão ser conduzidos, contribuindo para o manejo efetivo da sobrecarga de cuidadores familiares de idosos dependentes em áreas com vulnerabilidade socioambiental.

\section{Agradecimentos}

À Elyne Montenegro Engstrom e Edinilsa Ramos Souza que apoiaram a realização do estudo. À Secretaria Municipal de Saúde do Rio de Janeiro pelo financiamento. 


\section{Referências}

1. World Health Organization. World report on disability. Geneva: World Health Organization; 2011.

2. Garbin CA, Sumida DH, Moimaz SA, do Prado RL, Silva MM. Aging by the perspective of elderly caregivers. Ciênc Saúde Coletiva 2010; 15:2941-8.

3. Caldas CP. Envelhecimento com dependência: responsabilidades e demandas da família. Cad Saúde Pública 2003; 19:773-81.

4. Oliveira DC, D'Elboux MJ. Estudos nacionais sobre cuidadores familiares de idosos: revisão integrativa. Rev Bras Enferm 2012; 65:829-38.

5. Rodakowski J, Skidmore ER, Rogers JC, Schulz R. Role of social support in predicting caregiver burden. Arch Phys Med Rehabil 2012; 93:2229-36.

6. Gratão ACM, Talmelli LFS, Figueiredo LC, Rosset I, Freitas CP, Rodrigues RAP. Dependência funcional de idosos e a sobrecarga do cuidador. Rev Esc Enferm USP 2013; 47:137-44.

7. Nardi, EFR, Sawada NO, Santos JLF. The association between the functional incapacity of the older adult and the family caregiver's burden. Rev Latinoam Enferm 2013; 21:1096-103.

8. Seima MD, Lenardt MH. A sobrecarga do cuidador familiar de idoso com Alzheimer. Textos Contextos (Porto Alegre) 2011; 10:388-98.

9. Yamashita $\mathrm{CH}$, Amendola F, Gaspar JC, Alvarenga MRM, Oliveira MAC. Associação entre o apoio social e o perfil de cuidadores familiares de pacientes com incapacidades e dependência. Rev Esc Enferm USP 2013; 47:1359-66.

10. Loureiro LSN, Fernandes MGM, Marques S, Nóbrega MML, Rodrigues RAP. Burden in family caregivers of the elderly: prevalence and association with characteristics of the elderly and the caregivers. Rev Esc Enferm USP 2013; 47:1129-36.

11. Johannesen M, Logiudice D. Elder abuse: a systematic review of risk factors in community-dwelling elders. Age Ageing 2013; 42:292-8.

12. Cooper C, Selwood A, Blanchard M, Walker Z, Blizard R, Livingston G. Abuse of people with dementia by family carers: representative cross sectional survey. BMJ 2009; 338:b155.

13. Moraes CL, Apratto Júnior PC, Reichenheim ME. Rompendo o silêncio e suas barreiras: um inquérito domiciliar sobre a violência doméstica contra idosos em área de abrangência do Programa Médico de Família de Niterói, Rio de Janeiro, Brasil. Cad Saúde Pública 2008; 24:2289-300.

14. Duque AM, Leal MCC, Marques APO, Eskinazi FMV, Duque AM. Violência contra idosos no ambiente doméstico: prevalência e fatores associados (Recife/ PE). Ciênc Saúde Coletiva 2012; 17:2199-208.

15. Rospenda KM, Minich LM, Milner LA, Richman JA. Caregiver burden and alcohol use in a community sample. J Addict Dis 2010; 29:314-24.

16. Shye D, Mullooly JP, Freeborn DK, Pope CR. Gender differences in the relationship between social network support and mortality: a longitudinal study of an elderly cohort. Soc Sci Med 1995; 41:935-47.

17. Sherbourne CD, Stewart AL. The MOS social support survey. Soc Sci Med 1991; 32:705-14.
18. Domínguez Guedea MT, Damacena FA, Montiel Carbajal MM, Marcobich PO, Álvarez Hernández G, Valdéz Lizárraga L, et al. Necessidades de apoio social em cuidadores de familiares idosos mexicanos. Psicol Soc (Impr.) 2009; 21:242-9.

19. Robinson-Whelen S, Tada Y, MacCallum RC, McGuire L, Kiecolt-Glaser JK. Long-term caregiving: what happens when it ends? J Abnorm Psychol 2001; 110:573-84

20. Schütz G, Pivetta F, Engstron E. Contexto do TEIAS -Escola Manguinhos. In: Pivetta F, Carvalho MAP, organizadores. O Território Integrado de Atenção a Saúde em Manguinhos: todos somos aprendizes! Rio de Janeiro: Escola Nacional de Saúde Pública Sergio Arouca, Fundação Oswaldo Cruz; 2012. p. 25-37.

21. Souza ER, Ribeiro AP, Atie S, Souza AC, Marques CC. Rede de proteção aos idosos do Rio de Janeiro: um direito a ser conquistado. Ciênc Saúde Coletiva 2008; 13:1153-63.

22. Katz S, Ford AB, Moskowitz RW, Jackson BA, Jaffe MW. Studies of illness in the aged. The Index of ADL: a standardized measure of biological and psychosocial function. JAMA 1963; 185:914-9.

23. Lino VT, Pereira SR, Camacho LA, Ribeiro Filho ST, Buksman S. Adaptação transcultural da Escala de Independência em Atividades da Vida Diária (Escala de Katz). Cad Saúde Pública 2008; 24:103-12.

24. Folstein MF, Folstein SE, McHugh PR. "Mini-mental state": a practical method for grading the cognitive state of patients for the clinician. J Psychiatr Res 1975; 12:189-98.

25. Almeida OP. Mini Exame do Estado Mental e o diagnóstico de demência no Brasil. Arq Neuropsiquiatr 1998; 56:605-12.

26. Bertolucci PH, Brucki SM, Campacci SR, Juliano Y. O Mini-Exame do Estado Mental em uma população geral: impacto da escolaridade. Arq Neuropsiquiatr 1994; 52:1-7.

27. Alexopoulos GS, Abrams RC, Young RC, Shamoian CA. Cornell Scale for Depression in Dementia. Biol Psychiatry 1988; 23:271-84.

28. Carthery-Goulart MT, Areza-Fegyveres R, Schultz RR, Okamoto I, Caramelli P, Bertolucci AHF, et al. Versão brasileira da Escala Cornell de Depressão em Demência (Cornell Depression Scale in Dementia). Arq Neuropsiquiatr 2007; 65:912-5.

29. Yesavage JA, Brink TL, Rose TL, Lum O, Huang V, Adey M, et al. Development and validation of a geriatric depression screening scale: a preliminary report. J Psychiatr Res 1982-1983; 17:37-49.

30. Paradela EM, Lourenço RA, Veras RP. Validation of geriatric depression scale in a general outpatient clinic. Rev Saúde Pública 2005; 39:918-23.

31. Zarit SH, Orr NK, Zarit JM. Families under stress: caring for the patients with Alzheimer's disease and other related disorders. New York: New York University Press; 1985.

32. Scazufca M, Menezes PR, Almeida OP. Caregiver burden in an elderly population with depression in São Paulo, Brazil. Soc Psychiatry Psychiatr Epidemiol 2002; 37:416-22. 
33. Mayfield D, McLeod G, Hall P. The CAGE questionnaire: validation of a new alcoholism screening instrument. Am J Psychiatry 1974; 131:1121-3.

34. Masur J, Capriglione MJ, Monteiro MG, Jorge MR. Detecção precoce do alcoolismo em clínica médica através do questionário CAGE: utilidade e limitações. J Bras Psiquiatr 1985; 34:31-4.

35. Griep RH, Chor D, Faerstein E, Werneck GL, Lopes CS. Validade de constructo de escala de apoio social do Medical Outcomes Study adaptada para o português no Estudo Pró-Saúde. Cad Saúde Pública 2005; 21:703-14.

36. Reis M, Nahmiash D. Validation of the Caregiver Abuse Screen (CASE). Can J Aging 1995; 14 Suppl 2:45-60.

37. Paixão CM, Reichenheim ME, Moraes CL, Coutinho ES, Veras RP. Adaptação transcultural para o Brasil do instrumento Caregiver Abuse Screen (CASE) para detecção de violência de cuidadores contra idosos. Cad Saúde Pública 2007; 23:2013-22.

38. Abreu MNS, Siqueira AL. Regressão logística ordinal em estudos epidemiológicos. Rev Saúde Pública 2009; 43:183-94.

39. Loureiro LES, Fernandes M, Nóbrega MM, Rodrigues RA. Sobrecarga em cuidadores familiares de idosos: associação com características do idoso e demanda de cuidado. Rev Bras Enferm 2014; 67:227-32.

40. Viana MC, Gruber MJ, Shahly V, Alhamzawi A, Alonso J, Andrade LH, et al. Family burden related to mental and physical disorders in the world: results from the WHO World Mental Health surveys. Rev Bras Psiquiatr 2013; 35:115-25.

41. Cassis SV, Karnakis T, Moraes TA, Curiati JA, Quadrante AC, Magaldi RM. Correlation between burden on caregiver and clinical characteristics of patients with dementia. Rev Assoc Méd Bras 2007; 53:497-501.

42. Moraes SR, Silva LS. An evaluation of the burden of Alzheimer patients on family caregivers. Cad Saúde Pública 2009; 25:1807-15.

43. Castro-de-Araújo LF, Barcelos-Ferreira R, Martins CB, Bottino CM. Depressive morbidity among elderly individuals who are hospitalized, reside at longterm care facilities, and are under outpatient care in Brazil: a meta-analysis. Rev Bras Psiquiatr 2013; 35:201-7.
44. Silva SA, Scazufca M, Menezes PR. Population impact of depression on functional disability in elderly: results from "São Paulo Ageing \& Health Study" (SPAH). Eur Arch Psychiatry Clin Neurosci 2013; 263:153-8.

45. Hybels CF, Pieper CF, Blazer DG, Fillenbaum GG, Steffens DC. Trajectories of mobility and IADL function in older patients diagnosed with major depression. Int J Geriatr Psychiatry 2010; 25:74-81.

46. Ostacher MJ, Nierenberg AA, Iosifescu DV, Eidelman P, Lund HG, Ametrano RM, et al. Correlates of subjective and objective burden among caregivers of patients with bipolar disorder. Acta Psychiatr Scand 2008; 118:49-56.

47. Zivin K, Wharton T, Rostant O. The economic, public health, and caregiver burden of late-life depression. Psychiatr Clin North Am 2013; 36:631-9.

48. Adelman RD, Tmanova LL, Delgado D, Dion S, Lachs MS. Caregiver burden: a clinical review. JAMA 2014; 311:1052-60.

49. Drentea P, Clay OJ, Roth DL, Mittelman MS. Predictors of improvement in social support: five-year effects of a structured intervention for caregivers of spouses with Alzheimer's disease. Soc Sci Med 2006; 63:957-67.

50. Tomomitsu MR, Perracini MR, Neri AL. Fatores associados à satisfação com a vida em idosos cuidadores e não cuidadores. Ciênc Saúde Coletiva 2014; 19:3429-40.

51. Laranjeira R, Pinsky I, Sanches M, Zaleski M, Caetano R. Alcohol use patterns among Brazilian adults. Rev Bras Psiquiatr 2010; 32:231-41.

52. Galduróz JC, Carlini EA. Use of alcohol among the inhabitants of the 107 largest cities in Brazil, 2001. Braz J Med Biol Res 2007; 40:367-75.

53. Vitaliano PP, Zhang J, Scanlan JM. Is caregiving hazardous to one's physical health? A meta-analysis. Psychol Bull 2003; 129:946-72.

54. National Alliance for Caregiving; American Association of Retired Persons. Caregiving in the USA. http://www.caregiving.org/data/Caregiving_in_ the_US_2009_full_report.pdf (acessado em 10/ Mar/2015). 


\section{Abstract}

Population aging has led to increased dependency and overburden of family caregivers of dependent elderly. The aim was to verify prevalence of family caregivers overburden and associated factors in a poor and violent area of Rio de Janeiro, Brazil. This was a cross-sectional study of 140 elderly and family caregivers, focusing on social support, abuse, cohabitation, and family caregivers overburden, in addition to dependency, cognitive decline, and depression in the elderly. Multiple logistic models were constructed to explain family caregivers overburden. The following characteristics of the elderly were associated with family caregivers overburden: age $(O R=0.94 ; p<0.002)$, depression $(O R=2.59 ; p<0.005)$, and cognitive decline (OR = 3.19; $p<0.03)$. As for family caregivers characteristics, only social support remained relevant $(O R=2.35 ; p<0.005)$. In conclusion, investigating and treating depression and dementia in the elderly and promoting support for their caregivers can contribute to the effective management of family care givers overburden and improve quality of care for both.

Caregivers; Frail Elderly; Social Support

\section{Reumen}

El envejecimiento poblacional ocasionó un aumento de la dependencia y de la sobrecarga de cuidadores familiares de ancianos dependientes. El objetivo fue verificar la prevalencia y los factores asociados a la sobrecarga de cuidadores familiares en una región pobre y violenta en Río de Janeiro, Brasil. Se realizó un estudio transversal con 140 ancianos y cuidadores familiares para investigar: apoyo social, malos tratos, cohabita ción y sobrecarga en el cuidadores familiares; además de dependencia, declive cognitivo, depresión, en el an ciano. Se construyeron modelos logísticos múltiples con la esperanza de explicar la sobrecarga del cuidadores familiares. Las siguientes características de los ancia nos se asociaron a la sobrecarga: edad (OR $=0,94 ; p<$ $0,002)$, depresión $(O R=2,59 ; p<0,005) y$ declive cognitivo $(O R=3,19 ; p<0,03)$. En relación con los factores del cuidadores familiares, sólo el apoyo social mantuvo relevancia $(O R=2,35 ; p<0,005)$. Investigar y tratar la depresión y demencia en ancianos, así como proveer apoyo a sus cuidadores, puede contribuir al manejo efectivo de la sobrecarga de cuidadores familiares, mejorando la calidad del cuidado y la salud de ambos.

Cuidadores; Anciano Frágil; Apoyo Social
Recebido em 14/Abr/2015

Versão final reapresentada em 07/Ago/2015 Aprovado em 22/Fev/2016 